YITP-97-15

hep-th/9704010

\title{
Higher-dimensional WZW Model on Kähler Manifold and Toroidal Lie Algebra
}

\author{
Takeo Inami, Hiroaki Kanno* and Tatsuya Ueno \\ Yukawa Institute for Theoretical Physics \\ Kyoto University, Kyoto 606-01, Japan \\ and \\ Department of Mathematics, Faculty of Science* \\ Hiroshima University, Higashi-Hiroshima 739, Japan
}

\begin{abstract}
We construct a generalization of the two-dimensional Wess-Zumino-Witten model on a $2 n$-dimensional Kähler manifold as a group-valued non-linear sigma model with an anomaly term containing the Kähler form. The model is shown to have an infinitedimensional symmetry which generates an $n$-toroidal Lie algebra. The classical equation of motion turns out to be the Donaldson-Uhlenbeck-Yau equation, which is a $2 n$ dimensional generalization of the self-dual Yang-Mills equation.
\end{abstract}




\section{Introduction}

There has recently been a renewed interest in field theories and supergravity theories in diverse dimensions. They are believed to play the role of effective field theories of the conjectured ultimate theory of unification, e.g. some sort of superstring or supermembrane theory. Higher-dimensional field theories also deserve studying on their own right. It is an interesting question to find the integrable structure of the theories, which makes them solvable and leads to some good properties, e.g. their fine ultraviolet behaviour. Apparently the ultraviolet behaviour becomes worse as the space-time dimension increases, unless miraculous cancellation of divergences occurs due to large symmetry of the theories.

In the past, various attempts have been pursued to construct higher-dimensional integrable field theories [1], albeit without much success. One approach to find such theories is to generalize 2D integrable field theories, respecting their good properties on the integrability. Recently the study of a 4D analogue of the 2D Wess-ZuminoWitten (WZW) model has revealed a few remarkable properties of the model [2], [3]. The model, which we refer to as 4D Kähler WZW (KWZW) model, has an infinitedimensional (anti-)holomorphic symmetry [2] and is solvable in its algebraic sector [3]. It has also been shown that the model is one-loop on-shell finite in spite of the apparent non-renormalizability by power counting [3], [四].

In [5], we have clarified that the infinite-dimensional symmetry generates a current algebra identified with a two-toroidal Lie algebra, a central extension of the two-loop algebra, which a few mathematicians have recently begun to study as a possible extension of the affine Kac-Moody algebra [6]. It is interesting and natural to ask whether a higherdimensional generalization of the 2D WZW model extends to general $2 n$ dimensions.

In this paper, we will construct a $2 n$-dimensional analogue of the WZW model, which we call $2 n \mathrm{D}$ KWZW model since it is defined on a Kähler manifold. The model is a groupvalued non-linear sigma model (NLSM) with an addition of an anomaly term containing the $(n-1)$-th power of the Kähler form $\omega$. We will show that the $2 n \mathrm{D}$ KWZW model has 
a higher-dimensional generalization of many properties of the 2D WZW model, e.g. it has an infinite-dimensional symmetry generating an $n$-toroidal Lie algebra. The classical equation of motion of the model turns out to be the Donaldson-Uhlenbeck-Yau equation, which suggests a close relationship of the model to complex and algebraic geometry.

\section{$2 \quad 2 n \mathrm{D}$ KWZW model}

Let us consider a NLSM with torsion defined on a $2 n \mathrm{D}$ Riemannian manifold $X_{2 n}$. The basic field in the model is a mapping $\phi^{a}(x)$ from $X_{2 n}$ to a target space $\mathcal{T}$ with a metric $g_{a b}$ and a two-form $b=b_{a b} d \phi^{a} \wedge d \phi^{b}$. The most general form of the action which contains only second-order derivatives of $\phi^{a}(x)$ and is invariant under the reparametrizations of $X_{2 n}$ and $\mathcal{T}$, is written as, in differential form,

$$
S=\frac{f_{\pi}^{2 n-2}}{4 \pi} \int_{X_{2 n}} d \phi^{a} \wedge * d \phi^{b} g_{a b}(\phi)+\frac{\gamma}{4 \pi} \int_{X_{2 n}} \Theta_{2(n-1)} \wedge d \phi^{a} \wedge d \phi^{b} b_{a b}(\phi)
$$

where $\Theta_{2(n-1)}$ is a $2(n-1)$-form on $X_{2 n}$. Our convention is that the coupling constant $f_{\pi}$ has the mass dimension +1 , while all differential forms and the parameter $\gamma$ are dimensionless.

Let us assume the space-time $X_{2 n}$ is a Kähler manifold and $\Theta_{2(n-1)}$ is the $(n-1)$-ple product of a closed Kähler form $\omega$,

$$
\Theta_{2(n-1)}=\omega^{n-1}, \quad \omega=\frac{i}{2} f_{\pi}^{2} h_{\alpha \bar{\beta}} d z^{\alpha} \wedge d \bar{z}^{\beta}
$$

where $h_{\alpha \bar{\beta}}(\alpha, \bar{\beta}=1,2)$ is a Kähler metric on $X_{2 n}$. The target space $\mathcal{T}$ is assumed to be a group manifold $G$. Then vielveins $V_{a}^{i}(\phi)$ defined from $g_{a b}=V_{a}^{i} V_{b}^{j} \delta_{i j}$ obey the Maurer-Cartan equation,

$$
\partial_{a} V_{b}^{i}-\partial_{b} V_{a}^{i}-2 f^{i j k} V_{a}^{j} V_{b}^{k}=0
$$

This pure-gauge condition gives $V_{a}^{i} d \phi^{a} T^{i}=-\frac{i}{2} g^{-1} d g$, with generators $T^{i}$ of the Lie

algebra associated with the group $G ;\left[T^{i}, T^{j}\right]=i f^{i j k} T^{k}, \operatorname{Tr} T^{i} T^{j}=\frac{1}{2} \delta^{i j}$. Using it and 
(2), the first term in the action (1) is written as

$$
f_{\pi}^{2 n-2} \int_{X_{2 n}} d \phi^{a} \wedge * d \phi^{b} g_{a b}=-\frac{2^{(n-1)} i}{(n-1) !} \int_{X_{2 n}} \omega^{n-1} \wedge \operatorname{Tr} g^{-1} \partial g \wedge g^{-1} \bar{\partial} g
$$

where $\partial=\left(\partial / \partial z^{\alpha}\right) d z^{\alpha}\left(\bar{\partial}=\left(\partial / \partial \bar{z}^{\beta}\right) d \bar{z}^{\beta}\right)$. To re-express the second term in (1) , we introduce a $(2 n+1) \mathrm{D}$ manifold $X_{2 n+1}=X_{2 n} \times[0,1]$

$$
\begin{aligned}
\int_{X_{2 n}} \omega^{n-1} \wedge d \phi^{a} \wedge d \phi^{b} b_{a b} & =\frac{2}{3} \int_{X_{2 n+1}} \omega^{n-1} \wedge d \phi^{a} \wedge d \phi^{b} \wedge d \phi^{c} H_{a b c} \\
& =\frac{1}{3} \int_{X_{2 n+1}} \omega^{n-1} \wedge \operatorname{Tr}\left(g^{-1} d g\right)^{3},
\end{aligned}
$$

where $H_{a b c}=\frac{3}{2} \partial_{[a} b_{b c]}$ is the torsion on the manifold $G$ and is expressed by the structure constant $f_{i j k}$ as $H_{a b c}=V_{a}^{i} V_{b}^{j} V_{c}^{k} f_{i j k}$.

We set the value of the parameter $\gamma$ as

$$
\gamma=\frac{2^{n-1} i}{(n-1) !}
$$

for which a symmetry enhancement in the model takes place, as will be shown below. This value will also appear in the one-loop finiteness condition of the model. Finally the action (1) becomes, after re-scaling $f_{\pi}^{2 n-2} \rightarrow i \gamma^{-1} f_{\pi}^{2 n-2}$,

$$
S=-\frac{i}{4 \pi} \int_{X_{2 n}} \omega^{n-1} \wedge \operatorname{Tr} g^{-1} \partial g \wedge g^{-1} \bar{\partial} g+\frac{i}{12 \pi} \int_{X_{2 n+1}} \omega^{n-1} \wedge \operatorname{Tr}\left(g^{-1} d g\right)^{3} .
$$

The choice of the $\gamma$ in (6) leads to the following identity, which is a $2 n \mathrm{D}$ analogue of the Polyakov-Wiegmann (PW) formula [7],

$$
S[g h]=S[g]+S[h]-\frac{i}{2 \pi} \int_{X_{2 n}} \omega^{n-1} \wedge \operatorname{Tr} g^{-1} \partial g \bar{\partial} h h^{-1} .
$$

From this formula, we see that the action is invariant under holomorphic right and antiholomorphic left symmetries, $g \rightarrow h_{L}\left(\bar{z}^{\beta}\right) g h_{R}\left(z^{\alpha}\right)$.

The equation of motion is given by

$$
\bar{\partial}\left(\omega^{n-1} \wedge g^{-1} \partial g\right)=0
$$

or equivalently,

$$
\partial\left(\omega^{n-1} \wedge \bar{\partial} g g^{-1}\right)=0
$$


From this equation of motion, we can identify conserved currents $J(\bar{J})$ corresponding to the above right (left) action symmetry as

$$
J=-\frac{i}{2 \pi} \omega^{n-1} \wedge g^{-1} \partial g, \quad \bar{J}=\frac{i}{2 \pi} \omega^{n-1} \wedge \bar{\partial} g g^{-1}
$$

\section{Donaldson-Uhlenbeck-Yau equation}

We will show that the equation of motion (19) or (10) is equivalent to the DonaldsonUhlenbeck-Yau (DUY) equation in a particular gauge. On a $2 n \mathrm{D}$ Kähler manifold, the curvature two-form $F$ of a gauge field is decomposed into $(2,0),(1,1)$ and $(0,2)$ components. Then the equations

$$
\begin{aligned}
& F_{\alpha \beta}=F_{\bar{\alpha} \bar{\beta}}=0, \\
& h^{\alpha \bar{\beta}} F_{\alpha \bar{\beta}}=0,
\end{aligned}
$$

are called the DUY equation [8]. Note that these equations can be derived from the action of the $(2 n+1)$ D Kähler Chern-Simons theory [2]. In algebraic geometry the moduli space of stable holomorphic vector bundles plays a significant role. The DUY equation provides a description of this moduli problem in the differential geometric language.

The first condition (12) means that the connection is holomorphic and the second (13) is equivalent to the stability of the holomorphic bundle in algebraic geometry [8]. The DUY equation also appears in the Calabi-Yau compactification of the heterotic string theory [9]. To keep $N=1$ supersymmetry after compactification to four dimensions, the connection of the gauge bundle over the internal Calabi-Yau space should satisfy the DUY equation.

The holomorphy condition (12) is solved by setting

$$
A_{\alpha}=h_{L}^{-1} \partial_{\alpha} h_{L}, \quad A_{\bar{\beta}}=h_{R} \partial_{\bar{\beta}} h_{R}^{-1}
$$

where $h_{L}$ and $h_{R}$ are $G^{\mathbf{C}}$-valued fields. We have complexified the gauge group and do not assume the hermiticity of the gauge field for a moment. Then introducing $g \equiv h_{L} h_{R}$, 
we can express the $(1,1)$ component of the curvature as

$$
F^{(1,1)}=h_{R} \bar{\partial}\left(g^{-1} \partial g\right) h_{R}^{-1}=-h_{L}^{-1} \partial\left(\bar{\partial} g g^{-1}\right) h_{L}
$$

Since the stability condition (13) can be written as

$$
\omega^{n-1} \wedge F^{(1,1)}=0
$$

we see that it reduces to the equation of motion of the KWZW model (9) or (10). Thus the $2 n \mathrm{D}$ KWZW model gives the $2 n \mathrm{D}$ DUY equation and hence classically describes the moduli space of stable holomorphic vector bundles over a $2 n \mathrm{D}$ Kähler manifold.

In four dimensions, the DUY equation reduces to the anti-self-duality (ASD) condition on a Kähler manifold. Hence the equation of motion of the 4D KWZW model is nothing but the ASD condition, which has been discussed by several authors. As we have seen above, the DUY equation is a natural higher-dimensional analogue of the ASD condition specific to four dimensions. More than a decade ago, Corrigan et al. proposed the following first-order system for a gauge field [10],

$$
F_{\mu \nu}=\frac{1}{2} T_{\mu \nu \rho \sigma} F^{\rho \sigma}
$$

as a higher-dimensional (anti-)self-duality condition, or the instanton equation.

In the dimension $D=4$, we can take $S O(4)$ invariant tensor $\epsilon_{\mu \nu \rho \sigma}$ as a totally antisymmetric fourth rank tensor $T_{\mu \nu \rho \sigma}$, which gives the well-known instanton equation. If $D>4, T_{\mu \nu \rho \sigma}$ is no longer invariant under $S O(D)$, a holonomy group of a generic orientable Riemannian manifold. However, if the holonomy group is reduced to a subgroup $G \subset S O(D)$, we have a chance to obtain a $G$-invariant tensor $T_{\mu \nu \rho \sigma}$ and to define an analogue of the instanton equation (17). This is the case for a $2 n \mathrm{D}$ Kähler manifold with the holonomy $U(n)$. For example, on a 6D Kähler manifold with complex coordinates $\left(z^{\alpha}, \bar{z}^{\alpha}\right)$, the equation (17) with the tensor

$$
T_{\mu \nu \rho \sigma}=\epsilon_{\mu \nu \rho \sigma 1 \overline{1}}+\epsilon_{\mu \nu \rho \sigma 2 \overline{2}}+\epsilon_{\mu \nu \rho \sigma 3 \overline{3}}
$$

gives the DUY equation, that is, the equation of motion of the $6 \mathrm{D}$ KWZW model. 
The classical integrability of the ASD equation can be seen by representing it in the Lax form. Possible higher-dimensional generalization of this first-order formulation of the ASD equation was considered by Ward [11]. Generally the DUY equation does not coincide with equations in [11] and does not seem to allow a Lax representation. However, in the next section, we will show that the KWZW model has an infinite-dimensional current algebra. We believe that the algebra suggests a kind of solvability in some sector of the model.

\section{4 n-toroidal Lie algebra}

To obtain a current algebra in the model, we start with the classical Poisson bracket (P.B.) in the light-cone frame. The P.B. is defined by finding the symplectic structure of the model. In the following, we assume that the space-time $X_{2 n}$ is flat; $h_{\alpha \bar{\alpha}}=\frac{1}{2}$, $(\alpha=1, \cdots, n)$ with the others zero, and use the notation $u=z^{1}, v^{a}=z^{a+1}(\bar{u}=$ $\left.\bar{z}^{1}, \bar{v}^{a}=\bar{z}^{a+1}\right),(a=1, \cdots, n-1)$. We take $\bar{u}$ as our time coordinate, while the space variables are denoted as $\mathbf{x}=\left(u, v^{a}, \bar{v}^{a}\right)$. In this light-cone frame, the action (7) is firstorder in time derivatives, that is, it is already in Hamiltonian form. Therefore, we can easily obtain the symplectic two-form $\Omega_{i j}\left(\mathbf{x}, \mathbf{x}^{\prime}\right)$ from the action,

$$
\Omega_{i j}\left(\mathbf{x}, \mathbf{x}^{\prime}\right)=\frac{\kappa}{2^{n}} \delta_{i j} \partial_{u} \delta\left(u-u^{\prime}\right) \delta^{2(n-1)}\left(v-v^{\prime}\right)
$$

where $\kappa=\frac{i^{n^{2}}}{2^{n} \pi} f_{\pi}^{2(n-1)}(n-1)$ ! and $\delta^{2(n-1)}\left(v-v^{\prime}\right)=\prod_{a=1}^{n-1} \delta\left(v^{a}-v^{\prime a}\right) \delta\left(\bar{v}^{a}-\bar{v}^{\prime a}\right)$. Henceforth, we take the values of the coordinates real by using an analytic continuation. The P.B. is defined using $\Omega^{i j}$, the inverse of the symplectic form,

$$
\begin{aligned}
{\left[f_{1}(\mathbf{x}), f_{2}\left(\mathbf{x}^{\prime}\right)\right]_{\text {P.B. }} } & =\int d \tilde{\mathbf{x}} d \tilde{\mathbf{x}}^{\prime} \delta f_{1}(\mathbf{x}) /\left(\delta g g^{-1}(\tilde{\mathbf{x}})\right)^{i} \Omega^{i j}\left(\tilde{\mathbf{x}}, \tilde{\mathbf{x}}^{\prime}\right) \delta f_{2}\left(\mathbf{x}^{\prime}\right) /\left(\delta g g^{-1}\left(\tilde{\mathbf{x}}^{\prime}\right)\right)^{j} \\
& =2^{n+1} \kappa^{-1} \int d \tilde{\mathbf{x}} \operatorname{Tr}\left(\delta f_{1}(\mathbf{x}) / \delta g g^{-1}(\tilde{\mathbf{x}}) \partial_{\tilde{u}}^{-1} \delta f_{2}\left(\mathbf{x}^{\prime}\right) / \delta g g^{-1}(\tilde{\mathbf{x}})\right)
\end{aligned}
$$

The components of the current $J$ are given from (11) as $J_{A}=\kappa g^{-1} \partial_{A} g,\left(A=u, v^{a}\right)$. Since $\bar{u}$ is our time coordinate, from the conservation laws, $J_{u}^{i}$ are generators of the 
holomorphic right action symmetry $g \rightarrow g h_{R}\left(z^{\alpha}\right)$. If we take the $u$ as the time coordinate instead, then $\bar{J}_{\bar{u}}^{i}$ become generators of another anti-holomorphic left action symmetry. The P.B.'s of currents are

$$
\begin{aligned}
& {\left[\operatorname{Tr}\left(X J_{A}\right)(\mathbf{x}), \operatorname{Tr}\left(Y J_{B}\right)\left(\mathbf{x}^{\prime}\right)\right]_{\text {P.B. }}} \\
& \quad=-2^{n-1} \kappa \operatorname{Tr}\left\{g X g^{-1}(\mathbf{x}) \partial_{A} \partial_{u}^{-1} \partial_{B}\left(g Y g^{-1}(\mathbf{x}) \delta\left(u-u^{\prime}\right) \delta^{2(n-1)}\left(v-v^{\prime}\right)\right)\right\}
\end{aligned}
$$

where $X$ and $Y$ are elements of the algebra associated with the group $G$. In particular, for $(A, B)=(u, u)$,

$$
\left[J_{u}^{i}(\mathbf{x}), J_{u}^{j}\left(\mathbf{x}^{\prime}\right)\right]_{\text {P.B. }}=2^{n} i f_{i j k} J_{u}^{k}(\mathbf{x}) \delta\left(\mathbf{x}-\mathbf{x}^{\prime}\right)-2^{n} \kappa \delta^{i j} \partial_{u} \delta\left(\mathbf{x}-\mathbf{x}^{\prime}\right)
$$

The current algebra (22) can be cast into a more familiar form by making the mode expansion. To this end, we compactify the space directions such that $u, v^{a}$ and $\bar{v}^{a}$ take values in $[0,2 \pi]$. The charge of the holomorphic right action transformation by the generators $J$ is then given by

$$
Q^{k}=\int_{0}^{2 \pi} d u \prod_{a=1}^{n-1} d v^{a} \epsilon_{k}(u, v) \tilde{J}^{k}, \quad \tilde{J}^{k}=\frac{1}{2^{n}} \int_{0}^{2 \pi} \prod_{a=1}^{n-1} d \bar{v}^{a} J_{u}^{k}(\mathbf{x}) .
$$

From the conservation laws, we see that $\tilde{J}^{k}$ are functions of $u$ and $v^{a}$ only; $\tilde{J}^{k}=\tilde{J}^{k}\left(u, v^{a}\right)$. We use the notation $\mathbf{z}=\left(u, v^{a}\right)$. Then the P.B.'s for $\tilde{J}^{i}(\mathbf{z})$ are written as

$$
\left[\tilde{J}^{i}(\mathbf{z}), \tilde{J}^{j}\left(\mathbf{z}^{\prime}\right)\right]_{\text {P.B. }}=i f_{i j k} \tilde{J}^{k}(\mathbf{z}) \delta\left(\mathbf{z}-\mathbf{z}^{\prime}\right)-\frac{(2 \pi)^{n-1}}{2^{n}} \kappa \delta^{i j} \partial_{u} \delta\left(\mathbf{z}-\mathbf{z}^{\prime}\right)
$$

The parameter functions $\epsilon_{k}(\mathbf{z})$ are defined on the $n$-torus and can be expanded as

$$
\epsilon_{k}(\mathbf{z})=\sum_{\mathbf{m}} \epsilon_{k}^{\mathbf{m}} e^{i \mathbf{m} \cdot \mathbf{z}}
$$

where $\mathbf{m}=\left(m_{1}, m_{2}, \cdots, m_{n}\right) \in \mathbf{Z}^{n}$ and $\mathbf{m} \cdot \mathbf{z}=m_{1} u+m_{2} v^{1}+\cdots+m_{n} v^{n-1}$. Correspondingly, the modes of the currents $\tilde{J}^{k}(\mathbf{z})$ are defined as

$$
Q^{k}=\sum_{\mathbf{m}} \epsilon_{k}^{\mathbf{m}} J_{\mathbf{m}}^{k}, \quad J_{\mathbf{m}}^{k}=\int_{0}^{2 \pi} d u \prod_{a=1}^{n-1} d v^{a} e^{i \mathbf{m} \cdot \mathbf{z}} \tilde{J}^{k}(\mathbf{z}) .
$$

Then from (24) we obtain

$$
\left[J_{\mathbf{n}}^{i}, J_{\mathbf{m}}^{j}\right]_{\text {P.B. }}=i f_{i j k} J_{\mathbf{n}+\mathbf{m}}^{k}+i \lambda_{1} n_{1} \delta^{i j} \delta_{\mathbf{n},-\mathbf{m}}, \quad \lambda_{1}=\frac{(2 \pi)^{2 n-1}}{2^{n}} \kappa
$$


This P.B. algebra is an infinite-dimensional (i.e. $\mathbf{m} \in \mathbf{Z}^{n}$ ) Lie algebra with central extension, which is called $n$-toroidal Lie algebra [6]. Note that the integration over $\bar{v}^{a}$ of the original currents $J_{u}^{i}(\mathbf{x})$ is performed in (23) to obtain the generators $J_{\mathbf{n}}^{i}$ of the toroidal algebra. This $(n-1)$-ple integration also ensures the decouping of holomorphic and anti-holomorphic sectors of the total algebra; $\left[J_{\mathbf{n}}^{i}, \bar{J}_{\mathbf{m}}^{j}\right]_{\text {P.B. }}=0$.

There appears only one center proportional to $\lambda_{1} n_{1}$ in the P.B. (27), which corresponds to the coordinate $u$. The reason for this is that we have chosen $\bar{u}$ as the time coordinate and defined the P.B. with respect to it. It is, however, known that the $n(\geq 2)$-toroidal Lie algebra is an infinite-dimensional central extension of the $n$-loop algebra unlike the $n=1$ (affine Kac-Moody) case [6]. Hence, other central terms may arise in (27) when we evaluate quantum corrections to the P.B. algebra.

\section{Discussion}

We have shown that the $2 n \mathrm{D}$ KWZW model gives the Donaldson-Uhlenbeck-Yau (DUY) equation, or equivalently a generalization of the anti-self-dual (ASD) condition, as the equation of motion and that the model has an infinite-dimensional symmetry generating the $n$-toroidal Lie algebra. A natural question is whether the symmetry leads to nice properties of the model, such as the integrability and the one-loop finiteness.

The equation of motion in the 4D case reduces to the ASD Yang-Mills equation. It has been argued that behind the ASD equation there exists another type of infinitedimensional symmetry of the loop algebra [12], which is different from our toroidal algebra symmety in a few points. One crucial difference is that the former gives an affine KacMoody algebra with no central extension, whereas our toroidal algebra has a central term, which is an inevitable consequence of the anomaly term in the KWZW model. In this sense, the former is an analogue of the (hidden) symmetry of the 2D principal chiral model, while our symmetry is a genuine extension of the affine Kac-Moody symmetry in the 2D WZW model. Another difference is that the toroidal algebra symmetry is valid 
not only at the level of equation of motion but also at the level of action. This is a great advantage in an attempt to quantizing the model.

The above good properties of the toroidal algebra symmetry hold in general $2 n$ dimensions. It is worth investigating the integrability of the KWZW model and clarifying the role of the $n$-toroidal algebra.

In four dimensions, the one-loop ultra-violet divergences, which haunt the NLSM, have been shown to cancel completely, partially due to the two-toroidal algebra symmetry. Whether the same cancellation of the divergences persists in general $2 n \mathrm{D}$ KWZW model is an interesting issue. We are currently investigating the one-loop ultra-violet behaviour of the model in six and eight dimensions. We have witnessed cancellation of many divergent terms, but have not completed to check whether all such terms are eliminated or not.

The 4D KWZW model appears in an approach to the theory of unification based on the $\mathrm{N}=(2,1)$ heterotic string [13]. There, the (anti-)self-dual gauge field is a mapping (M-brane) from a $(2+2) \mathrm{D}$ world-volume to a $(10+2) \mathrm{D}$ target space, and various superstrings and supermembrane are obtained by reducing the $(2+2) \mathrm{D}$ to $(1+1) \mathrm{D}$ and $(2+1) \mathrm{D}$, respectively. The KWZW model describes the world-volume dynamics of the M-brane and may play the same role as conformal field theory in string theory.

We are grateful to Yoshihisa Saito for useful discussions on toroidal Lie algebras. We also wish to thank Chuan-Sheng Xiong, who collaborated with us in the early stage of the present work. This work is supported partially by Grant in Aid of the Ministry of Education, Science and Culture. T. U. is supported by the Japan Society for the Promotion of Science, No. 6293. 


\section{References}

[1] A.M. Polyakov, Nucl. Phys. B164 (1979) 171.

J.L. Cardy, Nucl. Phys. B366 (1991) 403.

[2] V.P. Nair and J. Schiff, Phys. Lett. B246 (1990) 423; Nucl. Phys. B371 (1992) 329.

[3] A. Losev, G. Moore, N. Nekrasov and S. Shatashvili, Nucl. Phys. B46 (Proc. Suppl.) (1996) 130 .

[4] S.V. Ketov, Phys. Lett. B383 (1996) 390.

[5] T. Inami, H. Kanno, T. Ueno and C-S. Xiong, 'Two-toroidal Lie Algebra as Current Algebra of Four-dimensional Kähler WZW model', hep-th/9610187, to appear in Phys. Lett. B.

[6] R.V. Moody, S. Eswara Rao and T. Yokonuma, Geom. Dedicata 35 (1990) 283.

S. Eswara Rao and R.V. Moody, Commun. Math. Phys. 159 (1994) 239.

S. Berman and B. Cox, Pacific J. Math. 165 (1994) 239.

S. Berman and R.V. Moody, Invent. Math. 108 (1992) 323.

I.B. Frenkel and B.A. Khesin, Commun. Math. Phys. 178 (1996) 541.

Y. Saito, 'Quantum Toroidal Algebras and their Vertex Representations', q-alg/9611030.

Y. Billig, 'Principal Vertex Operator Representations for Toroidal Lie Algebras', hep-th/9703002.

[7] A.M. Polyakov and P.B. Wiegmann, Phys. Lett. B131 (1983) 121.

[8] S.K. Donaldson, Proc. London Math. Soc. (3) 50 (1985) 1.

K.K. Uhlenbeck and S.T. Yau, Commun. Pure Appl. Math. 39 (1986) S257; ibid. 42 (1987) 703.

[9] M.B. Green, J.H. Schwarz and E. Witten, Superstring Theory:2, Chap.15, Cambridge University Press (1987). 
[10] E. Corrigan, C. Devchand, D.B. Fairlie and J. Nuyts, Nucl. Phys. B214 (1983) 452.

[11] R.S. Ward, Nucl. Phys. B236 (1984) 381.

[12] L-L. Chau, M-L. Ge and Y-S. Wu, Phys. Rev. D25 (1982) 1086.

L. Dolan, Phys. Lett. B113 (1982) 387.

L-L. Chau, Y-S. Wu, Phys. Rev. D26 (1982) 3581.

L-L. Chau, M-L. Ge, A. Sinha and Y-S. Wu, Phys. Lett. B121 (1983) 391.

[13] D. Kutasov and E. Martinec, Nucl. Phys. B477 (1996) 652; 'M-branes and N=2 Strings', hep-th/9612102.

D. Kutasov, E. Martinec and M. O'Loughlin, Nucl. Phys. B477 (1996) 675. 